\title{
The first direct double neutron star merger detection: Implications for cosmic nucleosynthesis
}

\author{
S. Rosswog ${ }^{1}$, J. Sollerman ${ }^{1}$, U. Feindt ${ }^{2}$, A. Goobar ${ }^{2}$, O. Korobkin ${ }^{3}$, R. Wollaeger ${ }^{3}$, C. Fremling ${ }^{4}$, and M. M. Kasliwal ${ }^{4}$ \\ ${ }^{1}$ The Oskar Klein Centre, Department of Astronomy, AlbaNova, Stockholm University, SE-106 91 Stockholm, Sweden \\ e-mail: stephan.rosswog@astro.su.se \\ 2 The Oskar Klein Centre, Department of Physics, AlbaNova, Stockholm University, SE-106 91 Stockholm, Sweden \\ ${ }^{3}$ Los Alamos National Laboratory, Los Alamos, NM 87545, USA \\ ${ }^{4}$ Division of Physics, Mathematics and Astronomy, California Institute of Technology, Pasadena, CA 91125, USA
}

Received 17 October 2017 / Accepted 4 April 2018

\begin{abstract}
Context. The astrophysical $r$-process site where about half of the elements, heavier than iron are produced, has been a puzzle for several decades. Here we discuss the role of one of the leading ideas - neutron star mergers (NSMs) - in the light of the first direct detection of such an event in both gravitational $(\mathrm{GW})$ and electromagnetic $(\mathrm{EM})$ waves.

Aims. Our aim is to understand the implications of the first GW/EM observations of a NSM for cosmic nucleosynthesis.

Methods. We analyse bolometric and NIR lightcurves of the first detected double NSM and compare them to nuclear reaction networkbased macronova models.

Results. The slope of the bolometric lightcurve is consistent with the radioactive decay of neutron star ejecta with $Y_{e} \lesssim 0.3$ (but not larger), which provides strong evidence for an $r$-process origin of the electromagnetic emission. This rules out in particular "nickel winds" as major source of the emission. We find that the NIR lightcurves can be well fitted either with or without lanthanide-rich ejecta. Our limits on the ejecta mass together with estimated rates directly confirm earlier purely theoretical or indirect observational conclusions that double neutron star mergers are indeed a major site of cosmic nucleosynthesis. If the ejecta mass was typical, NSMs can easily produce all of the estimated Galactic $r$-process matter, and - depending on the real rate - potentially even more. This could be a hint that the event ejected a particularly large amount of mass, maybe due to a substantial difference between the component masses. This would be compatible with the mass limits obtained from the GW-observation.

Conclusions. The recent observations suggests that NSMs are responsible for a broad range of $r$-process nuclei and that they are at least a major, but likely the dominant $r$-process site in the Universe.
\end{abstract}

Key words. gravitational waves - nuclear reactions, nucleosynthesis, abundances - dense matter - radiation mechanisms: general

\section{Introduction}

Soon after the discovery of the first binary neutron star (PSR 1913+16; Hulse \& Taylor 1975) it became clear that gravitational wave $(\mathrm{GW})$ emission drives the binary system towards a final coalescence (Taylor \& Weisberg 1982). Lattimer \& Schramm (1974) speculated that neutron star debris from such an encounter could be a promising production site $^{1}$ for the heaviest elements formed via "rapid neutron capture" or " $r$-process" (Burbidge et al. 1957; Cameron 1957; Thielemann et al. 2011). With the techniques available at that time they could, however, only estimate the ejecta mass to " $\sim 0.05 \pm 0.05 M_{\mathrm{ns}}$ ". The $r$-process is responsible for about half of the elements heavier than iron, but until recently the dominant opinion was that corecollapse supernovae (CC SNe) must be the major production site. Eichler et al. (1989) discussed merging neutron star binaries as "central engines" for short gamma-ray bursts (sGRBs) and as $r$-process production sites. The first nucleosynthesis calculations based on 3D hydrodynamic merger simulations (Rosswog et al. 1999) showed that the neutron-rich matter that is dynamically ejected indeed produces - robustly and without any

1 For technical reasons they performed the analysis for a neutron star black hole system. fine-tuning $-r$-process nuclei up to and beyond the third $r$-process peak at nucleon numbers of $A=195$ (Rosswog et al. 1998; Freiburghaus et al. 1999b). They also showed that the ejecta are - if folded with estimated merger rates - enough to explain the amount of $r$-process material in the Galaxy.

A large number of subsequent studies (e.g. Roberts et al. 2011; Goriely et al. 2011; Wanajo \& Janka 2012; Korobkin et al. 2012), have investigated these so-called "dynamic ejecta" as $r$-process sites. Only more recently, it was realised that the extremely low electron fraction (= electron to nucleon ratio $=$ proton to nucleon ratio) ejecta $\left(Y_{e} \lesssim 0.1\right)$ are likely complemented by matter reaching $Y_{e} \sim 0.3$, e.g. by shock-heated material (Wanajo et al. 2014; Radice et al. 2016), neutrinodriven winds (Dessart et al. 2009; Perego et al. 2014b) or the unbinding of accretion torus material (e.g. Lee \& Ramirez-Ruiz 2007; Beloborodov 2008; Metzger et al. 2008; Fernandez \& Metzger 2013; Just et al. 2015; Ciolfi \& Siegel 2015; Martin et al. 2015; Siegel \& Metzger 2017). This unbound torus material can amount to $\sim 40 \%$ of the initial torus mass and - depending on the initial mass asymmetry - can actually dominate the ejecta. Geometrically, there is the tendency of the low- $Y_{e}$ matter to be concentrated towards the orbital plane, while $Y_{e}$ increases towards the polar remnant regions, see e.g. Figs. 14 and 15 in Perego et al. (2014b). 
While initially questioned (e.g. Argast et al. 2004), a number of recent studies (Matteucci et al. 2014; Mennekens \& Vanbeveren 2014; van de Voort et al. 2015; Shen et al. 2015) find compact binary mergers at least as suitable or even preferred over $\mathrm{CC} \mathrm{SNe}$ as the major $r$-process production site. One of the differences between the main alternatives is that (at least "ordinary") CC SNe occur $~ 1000$ more frequently than compact binary mergers and therefore have to deliver a correspondingly smaller amount of $r$-process elements per event to account for the cosmic inventory. There are, however, various lines of arguments that favour rare events with large ejecta masses over frequent occurrences with smaller ones. For example, the geochemical enrichment of ${ }^{244} \mathrm{Pu}$ (Wallner et al. 2015; Hotokezaka et al. 2015) and the observation of $r$-process enriched ultra-faint dwarf galaxies (Beniamini et al. 2016; Hansen et al. 2017) both argue in favour of rare events with high mass ejection. The inferred rates and ejecta masses agree well with what is expected from neutron star mergers (NSMs).

The most direct confirmation of compact binary mergers as $r$-process sites, however, is the detection of electromagnetic radiation from the radioactive decay of freshly synthesised $r$-process elements in the aftermath of a merger, a so-called "macronova" or "kilonova" (e.g. Li \& Paczyński 1998; Kulkarni 2005; Rosswog 2005; Metzger et al. 2010; Kasen et al. 2013; Tanaka \& Hotokezaka 2013). The most compelling previous evidence for such a macronova has been the detection of an infrared excess in the aftermath of a short GRB (130603B; Tanvir et al. 2013; Berger et al. 2013).

The situation changed fundamentally on August 17, 2017 with the first direct detection of GWs from a neutron star binary, GW 170817 by the LIGO-Virgo collaboration (LVC; Abbott et al. 2017d). The electromagnetic follow-up of GW170817 has been described in many papers (e.g. Abbot et al. 2017c; Kasliwal et al. 2017; Smartt et al. 2017), and includes the first detection in gamma-rays (Goldstein et al. 2017) only 1.7 s after GW170817, via the optical and near-infrared (NIR) discovery and monitoring of AT2017gfo (Abbot et al. 2017c) to the late onset of the radio emission (Hallinan et al. 2017). The object was initially surprisingly bright and blue compared to pre-discovery predictions, it was discovered at an absolute magnitude of -15.7 in the $i$ band (Coulter et al. 2017). The source quickly declined in the optical bands and over the next 3 weeks was observed to decline in the NIR, overall in agreement with the family of macronova models presented by e.g. Kasen et al. (2013), Tanaka \& Hotokezaka (2013), Rosswog et al. (2017), and Wollaeger et al. (2018). This detection marks the beginning of the long-awaited era in multi-messenger astronomy.

A large variety of facets and implications of this event have been discussed in the recent literature (e.g. Abbott et al. 2017a,b; Evans et al. 2017; Kasen et al. 2017; Levan et al. 2017; Tanvir et al. 2017; Margutti et al. 2017). In this paper we focus on the implications of this first discovery for the cosmic nucleosynthesis. We will in particular analyze bolometric and near-infrared lightcurves with respect to what they imply for the ejecta parameters and their nucleosynthesis. We discuss the relevance of the inferred ejecta amount for the Galactic $r$-process inventory and update our recent predictions (Rosswog et al. 2017) for the detectability of AT2017gf-like events.

\section{Bolometric lightcurve: a clue to $r$-process nucleosynthesis}

We have performed a number of calculations with the nuclear reaction network WinNet (Winteler 2012; Winteler et al. 2012) to explore how sensitive the nuclear heating rates are to the physical expansion conditions, which we set up as described in Rosswog et al. (2017; their Sect. 2.2). We ran a grid of 16 expansion models covering a broad parameter range $\left([v / c] \times\left[Y_{e}\right]=[0.1,0.2,0.3,0.4] \times[0.1,0.2,0.3,0.4]\right)$. To keep the parameter space manageable we fix the initial entropy to $15 k_{\mathrm{B}}$. This is reasonable since a) for very low $Y_{e}$-values the results are insensitive to the exact entropy-value (Freiburghaus et al. 1999a,b) for higher $Y_{e}$ cases detailed simulation studies find narrow distributions around this value (Perego et al. 2014b; Radice et al. 2016). For each case a power-law approximation for the nuclear heating rate (in $\operatorname{erg~g}^{-1} \mathrm{~s}^{-1}$ )

$\dot{q}=\dot{q}_{0}\left(\frac{t}{t_{0}}\right)^{\alpha}$

was determined from the network data (for $t>10^{-4} \mathrm{~d}$; at earlier times the heating rate is roughly constant), see Table 1 . We find that the power-law index $\alpha \approx-1.3$ for as long as $Y_{e} \lesssim 0.3$, consistent with earlier findings (Metzger et al. 2010; Korobkin et al. 2012; Hotokezaka et al. 2017). When $Y_{e}=0.4$ the heating rate drops off substantially faster, and the normalisation constant $\dot{q}_{0}$ is typically an order of magnitude lower $^{2}$. At early times when opacity effects are significant, diffusion can substantially affect the lightcurve shape. Once the ejecta are optically thin, and excess radiation produced earlier had time to escape, the lightcurve slope is determined by the heating rate from radioactive decay, modulo heating efficiency, $f_{\text {tot }}$. We compare the heating rate with the bolometric luminosity where we use data from the Kilonova catalog ${ }^{3}$. The bulk of the NIR data come from Smartt et al. (2017), Kasliwal et al. (2017), and Tanvir et al. (2017) as discussed in Villar et al. (2017), and additional $g$-band data mainly come from Pian et al. (2017), Arcavi et al. (2017), Coulter et al. (2017), and Cowperthwaite et al. (2017). We find that the slope of the bolometric luminosity (yellow filled circles) agrees excellently with the one of the nuclear heating rates $\dot{q}$, provided that the electron fraction $Y_{e} \lesssim 0.3$, but not larger. Along such low- $Y_{e}$ trajectories $r$-process elements are forged, see Fig. 2, and therefore the excellent agreement with the observed bolometric luminosity strongly suggests an $r$-process origin of the observed emission. As a further comparison, we also plot a trajectory with $Y_{e}=0.5$ which produces a substantial amount of nickel (marked with open polygons; same trajectory that produces the abundance pattern shown in the last panel of Fig. 2). This rules out a nickel wind as the primary source of bolometric luminosity.

To illustrate the impact of the electron fraction $Y_{e}$ on the resulting abundance pattern, we plot in Fig. 2 the results for typical ejecta conditions ( $s=15 \mathrm{k}_{\mathrm{B}}, v_{\mathrm{ej}}=0.25 \mathrm{c}$ ) where we systematically vary $Y_{e}$ from 0.05 to 0.5 . Below $Y_{e}^{\text {crit }} \approx 0.25$ "heavy" $r$-process including lanthanides up to and beyond the "platinum peak" at $A=195$ are produced, with a very robust abundance pattern for $Y_{e} \lesssim 0.15$. Above $Y_{e}^{\text {crit }} r$-process still occurs, but produces only light $r$-process elements $(A \lesssim 130)$. At the high $Y_{e}$-end substantial amounts of nickel are produced.

The efficiency with which released energy is translated into electromagnetic emission depends on the detailed decay products (Barnes et al. 2016), and varies from $\sim 0.7$ at 1 day to $\sim 0.3$ at 20 days, see Fig. 8 in Rosswog et al. (2017). Therefore, the net heating rates $\left(\dot{q} f_{\text {tot }}\right)$ decay slightly faster than the "naked"

\footnotetext{
2 Obviously, for this case a single power law is not a good approximation.

3 https://kilonova. space/ retreived on Jan. 18, see Guillochon et al. (2017).
} 


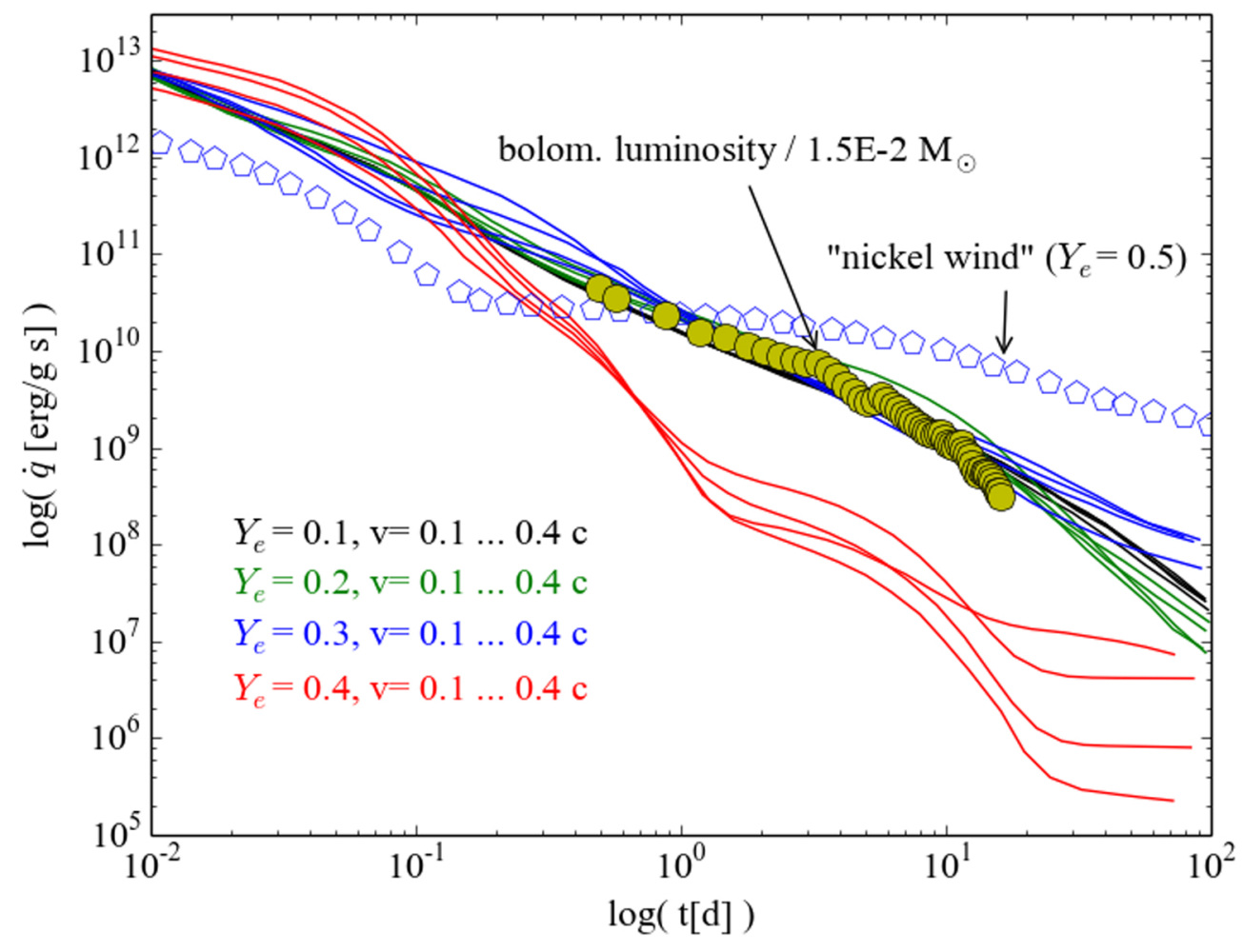

Fig. 1. Nuclear heating rates of the explored parameter space, colours label $Y_{e}$-values. Overlaid are bolometric luminosities computed following the description in Kasliwal et al. (2017) using updated photometry from https://kilonova.space (yellow circles). We show the total nuclear heating rate (luminosities divided by an ejecta mass of $1.5 \times 10^{-2} M_{\odot}$ ). Also shown is the heating rate of a wind with $Y_{e}=0.5$ that produces a substantial amount of nickel, see last panel in Fig. 2. The close agreement with $Y_{e} \lesssim 0.3$ strongly suggests the presence of substantial amounts of $r$-process matter. ones, though still in very good agreement with the observed bolometric lightcurve.

Assuming that $100 \%$ of the radioactive energy ends up in the observed emission places a lower limit on the ejected mass of

$m_{\mathrm{ej}}^{\min } \equiv \frac{L_{\mathrm{bol}}}{\dot{q}} \approx 1.5 \times 10^{-2} M_{\odot}$.

For a fixed set of nuclear physics ingredients this lower limit is robust. It has, however, been stressed by both Barnes et al. (2016) and Rosswog et al. (2017) that different nuclear mass models yield different amounts of trans-lead nuclei, the decays of which can substantially enhance the nuclear heating rate. For example, the results for the Finite-Range Droplet Model (FRDM; this mass model is used in WinNet; Moeller 1995) and the nuclear mass model of Duflo and Zuker (Duflo \& Zuker 1995; hereafter DZ) differed at time scales of about a day by a factor of $\sim 5$ in their net heating rates $\dot{q} f_{\text {tot }}$. Therefore, if a large fraction of the ejecta would have an electron fraction $<0.25$ and the nuclear heating would be close to the DZ-predictions, this mass limit could be smaller by a factor of $\sim 5$.

Since the bolometric light curve seems equally well fitted by all the models with electron fractions $Y_{e} \lesssim 0.3$, but only material with $Y_{e}<Y_{e}^{\text {crit }} \approx 0.25$ produces the third $r$-process peak, see Fig. 2, the bolometric luminosities alone are not conclusive regarding the ejecta composition. In particular it does not allow to infer whether lanthanides are present or whether the third $r$-process peak with elements such as platinum or gold is produced. For the purpose of illustration, we plot in Fig. 3 the resulting abundances for three trajectories. The first two yield an excellent fit to the slope of the bolometric light curve, but one $\left(Y_{e}=0.2, v=0.1 c\right)$ produces the full $r$-process range (but abundances below the second peak are produced only sub-dominantly) while the other $\left(Y_{e}=0.3, v=0.2 c\right)$ does not produce $r$-process beyond nucleon numbers $A>130$. For comparison we also show the abundances for $Y_{e}=0.4$ case which produces only elements up to $A \approx 90$.
Table 1. Coefficients for power-law fits for nuclear heating rates of the form $\dot{q}=\dot{q}_{0}\left(\frac{t}{t_{0}}\right)^{\alpha}$, where $t_{0}=1$ day.

\begin{tabular}{cccc}
\hline \hline$v[c]$ & $Y_{e}$ & $\dot{q}_{0}\left[10^{10} \mathrm{erg}\left(\mathrm{g} \mathrm{s}^{-1}\right]\right.$ & $\alpha$ \\
\hline 0.1 & 0.1 & 1.74 & -1.31 \\
& 0.2 & 2.14 & -1.28 \\
& 0.3 & 2.23 & -1.31 \\
& 0.4 & 0.234 & -1.66 \\
0.2 & 0.1 & 1.80 & -1.31 \\
& 0.2 & 1.75 & -1.31 \\
& 0.3 & 2.48 & -1.27 \\
& 0.4 & 0.140 & -1.77 \\
0.3 & 0.1 & 1.88 & -1.30 \\
& 0.2 & 1.67 & -1.31 \\
& 0.3 & 2.35 & -1.25 \\
& 0.4 & 0.104 & -1.81 \\
0.4 & 0.1 & 1.92 & -1.30 \\
& 0.2 & 1.73 & -1.30 \\
& 0.3 & 2.28 & -1.23 \\
& 0.4 & 0.289 & -1.64 \\
\hline
\end{tabular}

\section{Late near-infrared lightcurves}

The most conservative expectation prior to GW/EM170817 was a red EM-transient due to high-opacity ejecta peaking days after the GW-chirp (Kasen et al. 2013; Tanaka \& Hotokezaka 2013; Barnes et al. 2016; Rosswog et al. 2017; Wollaeger et al. 2018). Although the emergence of an additional blue component was discussed in theoretical work (Barnes \& Kasen 2013; Rosswog et al. 2014; Grossman et al. 2014; Metzger \& Fernandez 2014; Perego et al. 2014b; Martin et al. 2015; Fernandez et al. 2015; Kasen et al. 2015), the brightness of the blue optical transient (AT2017gfo) that was detected (Coulter et al. 2017) hours after the GW-chirp came as a surprise to most in the community. It 

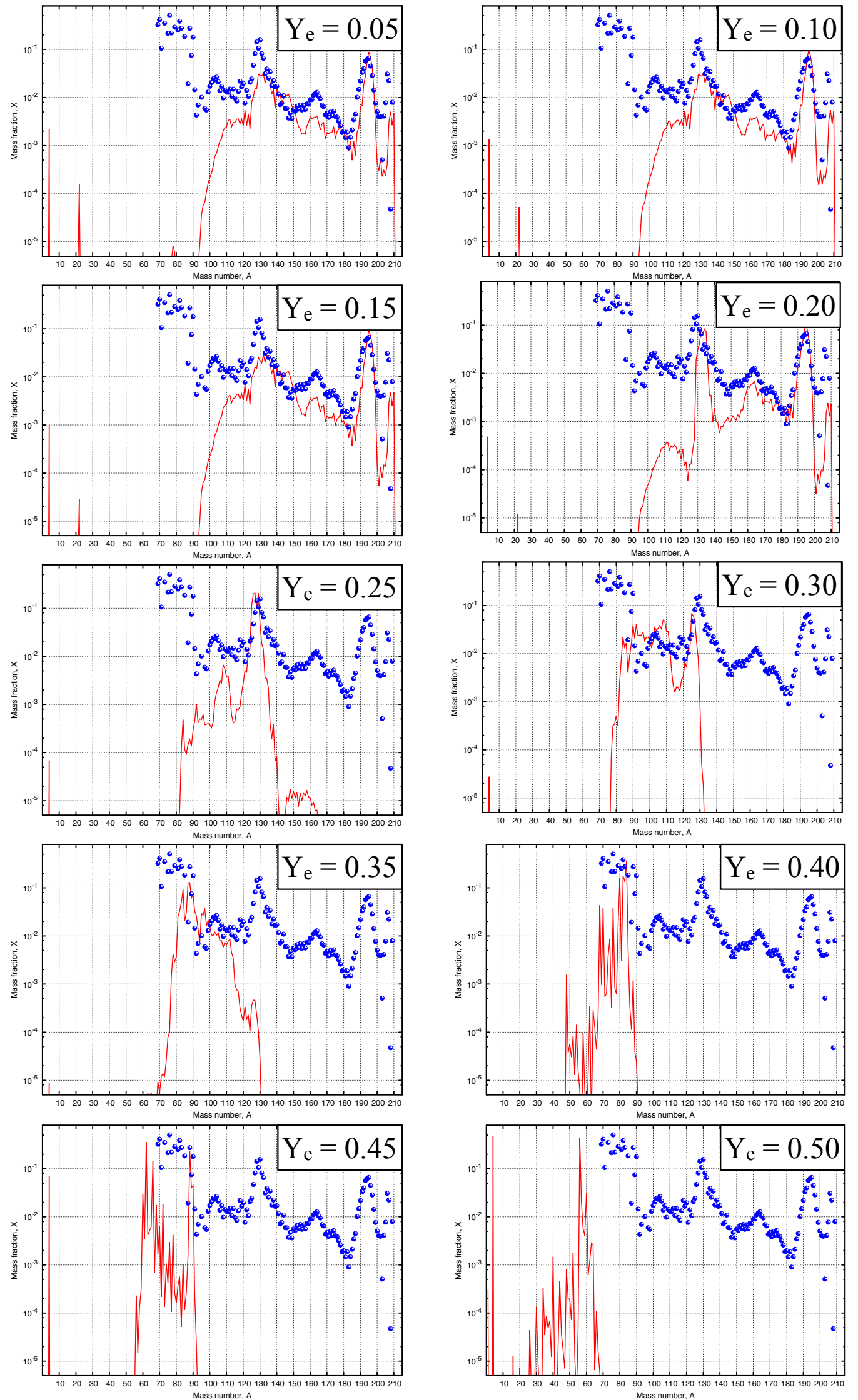

Fig. 2. Dependence of the nucleosynthesis on the electron fraction $Y_{e}$ (from 0.05 upper left to 0.5 lower right), for $s_{0}=15 k_{\mathrm{B}} / \mathrm{baryon}, v_{\mathrm{ej}}=0.25 \mathrm{c}$ and FRDM mass model. Red lines label the resulting abundances, blue symbols refer to the solar system $r$-process. Beyond $Y_{e}^{\text {crit }} \approx 0.25$ hardly any heavy elements beyond the second $r$-process peak $(A=130)$ are produced. 


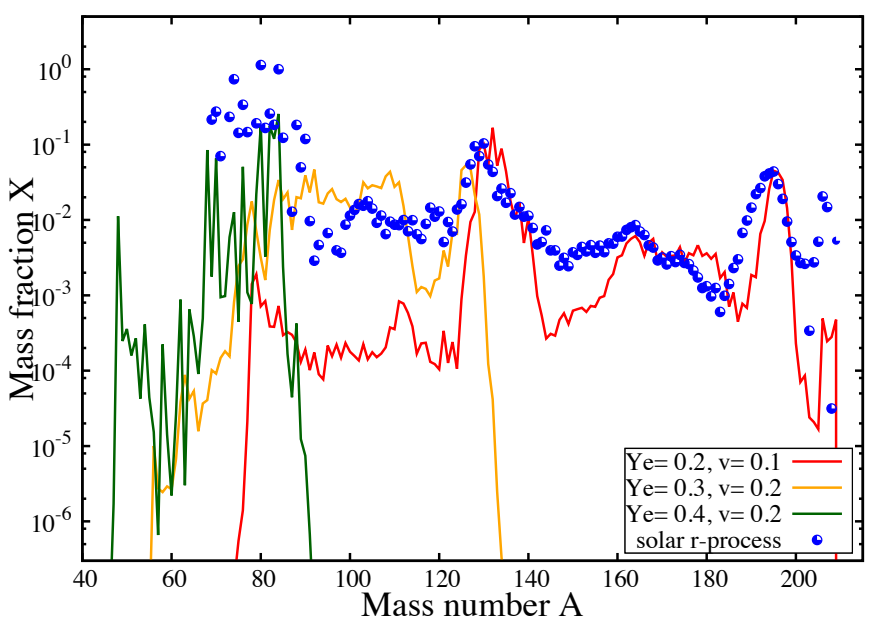

Fig. 3. Abundances for two cases (red and orange lines) that can both reproduce the bolometric luminosity evolution. While both produce $r$-process material, one case produces the third $r$-process peak $\left(Y_{e}=0.2\right.$ and $v=0.1 c)$, but the other $\left(Y_{e}=0.3\right.$ and $\left.v=0.2 c\right)$ does not. Thus, from the bolometric lightcurve alone the absence/presence of lanthanides cannot be inferred. For comparison, we also show a case (green line) with large $Y_{e}=0.4(v=0.2 c)$ that only produces elements with $A<90$. That case does not fit the bolometric luminosity.

can be explained by lower opacity material, potentially accelerated to mildly relativistic velocities through a GRB-jet producing a cocoon while plowing through previously ejected material (Kasliwal et al. 2017) or by a strong wind with moderately high electron fraction (Evans et al. 2017). This early blue component will not be discussed here.

Instead, we focus on the late NIR emission in the $\mathrm{J}-, \mathrm{H}$-, and $K$-bands. We have explored the parameter space in electron fraction, velocity, and ejected mass in more than 220 nuclear network based macronova simulations. For each model the initial conditions are set up as described in detail in Sect. 2.4.3 of Rosswog et al. (2017) and the nuclear heating history $\dot{q}(t)$ is calculated using the WinNet reaction network with the FRDM mass formula. We use time-dependent heating efficiencies $f_{\text {tot }}$ based on the work of Barnes et al. (2016) as calculated in Rosswog et al. (2017). Here we use the time-dependent averages of the FRDMcases explored in the latter work (their Fig. 8). We account for the uncertainty in the nuclear heating rate due to the $\alpha$-decay of trans-lead nuclei (as discussed in Sect. 2) in some experiments by enhancing the net heating rate of the FRDM results by a factor of 5 and refer to it as "DZ-type heating".

To extract the radiative signature we use a semianalytic eigenmode expansion formalism based on Pinto \& Eastman (2000). This semi-analytic approach has been shown to yield good agreement with more complex radiative transfer models and represents an improvement over the simpler model of Grossman et al. (2014) that we used in earlier work. Our approach is briefly summarized in Appendix A.

The NIR lightcurves alone leave some ambiguity as to what the exact ejecta parameters are, but they can be significantly constrained further if more data sets are taken into account. Interesting examples of NIR lightcurves are shown in Fig. 4. The low $Y_{e}$ is characteristic for the "tidal" component of dynamic ejecta that is ejected immediately during the merger at its original, very low electron fraction and produces substantial $r$-process contributions from $A \approx 100$ up to and beyond the platinum peak. The left panel shows a good fit of the NIR light curves for the case that the heating rate from the FRDM nuclear mass model is used $\left(m_{\mathrm{ej}}=0.06 M_{\odot}, Y_{e}=0.1, v_{\mathrm{ej}}=0.15 c\right.$, $\kappa=10 \mathrm{~cm}^{2} \mathrm{~g}^{-1}$ ). If instead DZ-type nuclear heating is used, our best parameters differ from the FRDM case $\left(m_{\mathrm{ej}}=0.006 M_{\odot}\right.$, $\left.Y_{e}=0.1, v_{\mathrm{ej}}=0.15 c, \quad \kappa=10 \mathrm{~cm}^{2} \mathrm{~g}^{-1}\right)$, and in particular substantially less mass is required.

Interestingly, the NIR late-time light curves do not necessarily prove the presence of either lanthanides or third $r$-process peak elements, although based on theoretical modelling their presence is certainly expected. It is also possible to obtain a good fit for an electron fraction $\left(Y_{e}=0.28\right)$ that is large enough to avoid the production of lanthanides and the third $r$-process peak and thus has a lower effective opacity $\left(\kappa=1 \mathrm{~cm}^{2} g^{-1}\right)$, see Fig. 5. The mass of $0.05 M_{\odot}$ could plausibly be ejected from a $\approx 0.13 M_{\odot}$ torus (assuming $40 \%$ ejection) and also the electron fraction is in the range expected for matter that has been exposed to a merger background neutrino field (Qian \& Woosley 1996; Rosswog et al. 2014; Perego et al. 2014b; Siegel \& Metzger 2017). Only the velocities are larger (by a factor of $\sim 2$ ) than what simulations (Fernandez \& Metzger 2013; Just et al. 2015) have found so far for unbound torus matter.

In order to see whether the comparison with another band can break the degeneracy between matter with and without lanthanides, we have added the $g$ band (green) to Figs. 4 and 5 . Based on this comparison alone, there would be a slight advantage for the lanthanide-free case. We would, however, consider it very unlikely that a merger starting out from cold, high-density $\beta$-equilibrium with $Y_{e} \approx 0.06$ manages to raise the electron fractions of all the ejecta beyond the critical value of $\approx 0.25$. To conclusively decide between the two cases may be beyond the capabilities of the current modelling (ours and in general). An obvious caveat is our use of constant gray opacities. In reality, opacities and the position of the photosphere are wavelength dependent. Another strong limitation stems from using only one value for electron fraction and velocity. This clearly is a strong simplifications and what has been observed is a superposition of distributions of physical conditions.

\section{Discussion}

The observation of GW170817 is a milestone. The first direct observation of a NSM and its coincident electromagnetic detection has finally proven two long-held suspicions, namely i) that such mergers are a source of short $\mathrm{GRBs}^{4}$ and - as we have demonstrated here - ii) it provides a first direct proof that their ejecta are a major source for the cosmic $r$-process nucleosynthesis.

We have explored the radioactive heating rate for a broad range of physical conditions and we find that the decline of the observed bolometric luminosity of AT2017gfo agrees very well with the decay produced by matter with $Y_{e} \lesssim 0.3$, but not larger. Such matter is subjected to the rapid-neutron capture process, see Fig. 2. The bolometric lightcurve rules out in particular nickel winds as the major source of the emission. This provides strong, direct observational evidence for NSMs being a major nucleosynthesis site and confirms earlier purely theoretical or indirect observational conclusions (Lattimer \& Schramm 1974; Rosswog et al. 1998, 1999; Freiburghaus et al. 1999b; Korobkin et al. 2012; Hotokezaka et al. 2015; Beniamini et al. 2016).

\footnotetext{
4 It is currently debated whether the event was a typical short GRB. While Kasliwal et al. (2017) argue that this was not a classical short burst GRB seen off-axis and interpret the emission instead in terms of a cocoon model, Lyman et al. (2018) find the late optical emission being consistent with the expectations from a structured jet that would - if seen on-axis - have been interpreted as a high-luminosity short GRB.
} 
$\mathrm{m}_{\mathrm{ej}}=0.06 \mathrm{M}_{\mathrm{sol}}, \mathrm{Y}_{\mathrm{e}}=0.1, \mathrm{v}_{\mathrm{ej}}=0.15 \mathrm{c}, \mathrm{\kappa}=10 \mathrm{~cm}^{2} / \mathrm{g} ; \mathrm{FRDM}, \mathrm{t}-$ dep. effic.

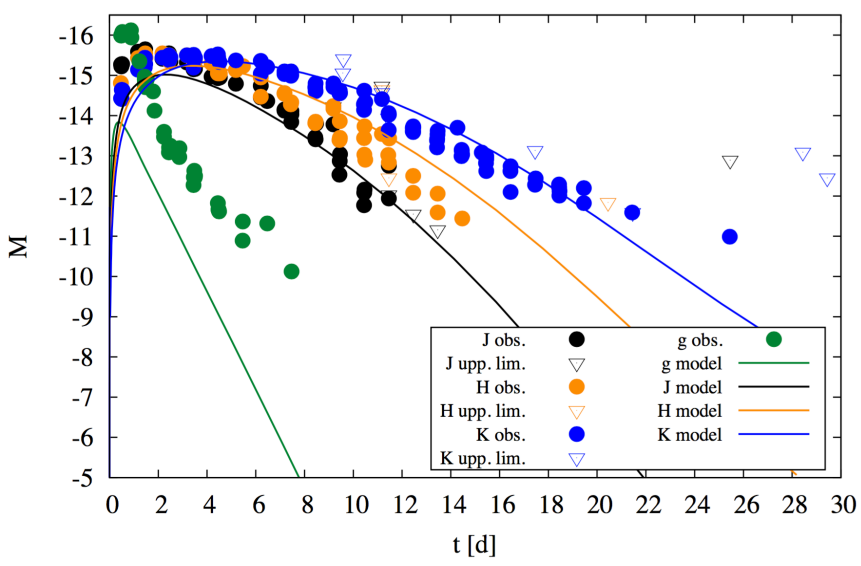

$\mathrm{m}_{\mathrm{ej}}=0.006 \mathrm{M}_{\mathrm{sol}}, \mathrm{Y}_{\mathrm{e}}=0.1, \mathrm{v}_{\mathrm{ej}}=0.25 \mathrm{c}, \kappa=10 \mathrm{~cm}^{2} / \mathrm{g} ; \mathrm{DZ}$, t-dep. effic.

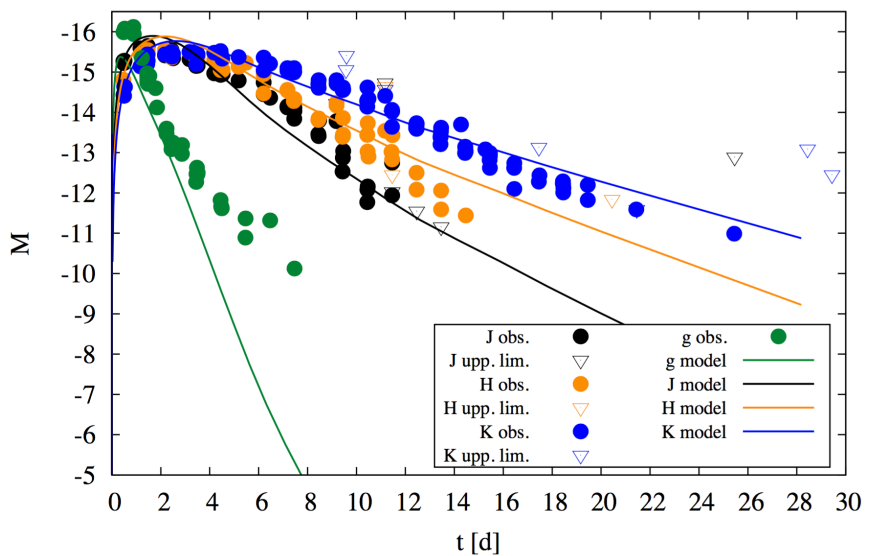

Fig. 4. Comparison of a low- $Y_{e}(=0.1)$ matter case, representative for a "tidal" dynamical ejecta, with NIR $J H K$-band observations (https://kilonova.space/kne/GW170817/). The detailed ejecta model parameters are shown at the top of each panel. The left panel uses heating according to the FRDM nuclear mass model, for the right panel a DZ-type heating rate has been employed (see Sect. 3 in the main text for a discussion).

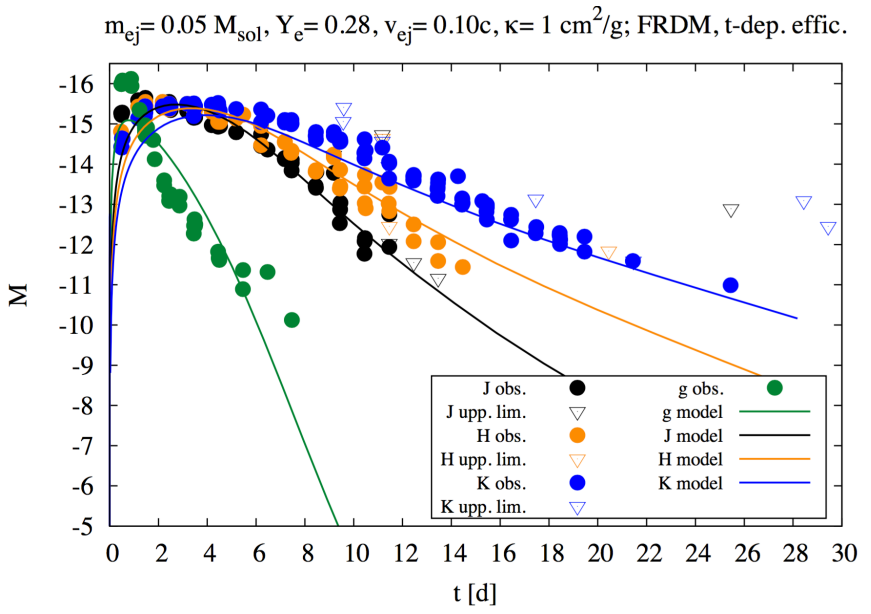

Fig. 5. Comparison for a model without lanthanides and third $r$-process peak ejecta. The detailed ejecta model parameters are shown at the top of the panel. For this calculation the heating rate of the FRDM nuclear mass model was used (see Sect. 3, main text for a discussion). The properties of this model are characteristic for matter unbound from an accretion torus.

Using nuclear network calculations employing the FRDM nuclear mass model, we derive a lower limit on the ejecta mass of $\approx 1.5 \times 10^{-2} M_{\odot}$ to explain the bolometric luminosity. Due to uncertainties in the nuclear physics far from stability, this limit could potentially be reduced by a factor of up to $\sim 5$. Even in this most pessimistic case the real ejecta amount would likely be $\sim 1 \%$ of a solar mass, which is a substantial amount in a cosmic nucleosynthesis context. Based on this first detected GW-event, the NSM rate (90\% conf.) is estimated as $320-4740 \mathrm{Gpc}^{-3} \mathrm{yr}^{-1}$ (Abbott et al. 2017d), compact object merger rate estimates based on SWIFT sGRB data point to $\sim 500-1500 \mathrm{Gpc}^{-3} \mathrm{yr}^{-1}$ (Petrillo et al. 2013) while recent population synthesis studies (Kim et al. 2015) estimate the rate ${ }^{5}$ as $244_{-162}^{+325} \mathrm{Gpc}^{-3} \mathrm{yr}^{-1}$, which means that within the rate uncertainties, NSMs can well produce all the $r$-process elements in the MW $\left(M_{r} \sim 19000 M_{\odot}\right.$; e.g.

5 We use the density of Milky Way equivalent galaxies of Abadie et al. (2010) to transform between different units.

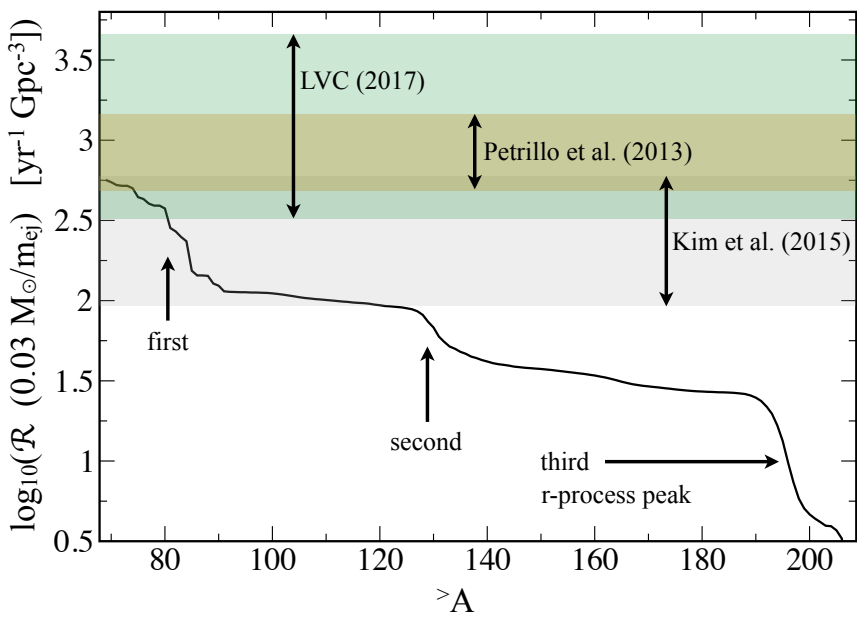

Fig. 6. Needed event rates, scaled to an ejecta mass of $0.03 M_{\odot}$, if NSNS mergers are to produce all $r$-process (in solar proportions) above a minimum nucleon number ${ }^{>} \mathrm{A}$ (solid black line). Also shown are the estimated rates (90\% conf.) for NSNS mergers from the population synthesis study of Kim et al. (2015), the sGRB rates based on Swift data from Petrillo et al. (2013) and the LVC estimate based on the first detected NSNS merger event.

Bauswein et al. 2014; Shen et al. 2015; Rosswog et al. 2017),

$$
M_{r} \sim 17000 M_{\odot}\left(\frac{\mathcal{R}_{\mathrm{NSNS}}}{500 \mathrm{Gpc}^{-3} \mathrm{yr}^{-1}}\right)\left(\frac{\bar{m}_{\mathrm{ej}}}{0.03 M_{\odot}}\right)\left(\frac{\tau_{\mathrm{gal}}}{1.3 \times 10^{10} \mathrm{yr}}\right) .
$$

Clearly, which rate is needed depends on which $r$-process elements are produced. In Fig. 6, we show as solid black line the required event rate (scaled to an ejecta mass of $0.03 M_{\odot}$ ) under the assumption that NSMs produce all $r$-process (in solar proportions) above a limiting nucleon number ${ }^{>} \mathrm{A}$. So if all $r$-process is produced in NSMs, an event rate of about $560\left(0.03 M_{\odot} / \bar{m}_{\mathrm{ej}}\right) \mathrm{yr}^{-1} \mathrm{Gpc}^{-3}$ is needed. If instead, NSMs should only produce $r$-process beyond the second peak $(A>130)$, a rate of only $70\left(0.03 M_{\odot} / \bar{m}_{\mathrm{ej}}\right) \mathrm{yr}^{-1} \mathrm{Gpc}^{-3}$ would suffice. The early blue emission observed in AT2017gfo, however, is most naturally explained with lower-opacity ejecta and therefore 
argues for the production of at least some lower-mass $r$-process material, which would also be consistent with recent theoretical studies (Wanajo et al. 2014; Perego et al. 2014b; Just et al. 2015; $\mathrm{Wu}$ et al. 2016). This could point to rates between the above two extremes. From the modelling of the NIR lightcurves alone it is not possible to distinguish between a pure high-opacity and pure low-opacity case (Figs. 4 and 5), but merger simulations indicate that at least some low- $Y_{e}$ matter is ejected and this is also consistent with the broad spectral features that have been observed (Kasliwal et al. 2017; Tanvir et al. 2017; Chornock et al. 2017). Therefore, we interpret this first event as strong evidence for a broad range of $r$-process nuclei being produced and not just - as thought until a few years ago - only $A>130$ material.

Based on the discussed numbers, NSMs could produce all the cosmic $r$-process without needing an additional production site. But within the uncertainties of rates/ejecta masses additional contributors are certainly possible. It has been argued (see e.g. the discussion in Thielemann et al. 2017) that an additional source of strong $r$-process would make it easier to explain the very large scatter of $[\mathrm{Eu} / \mathrm{Fe}]$ observed already at very low metallicities and the presence of so-called "actinide boost" stars (Lai et al. 2008) that have a fourfold enhancement of thorium and uranium relative to europium. As a potential additional strong $r$-process source core-collapse supernovae have been suggested (Winteler et al. 2012) that eject $r$-process matter in magnetohydrodynamial jets. Recent 3D MHD studies (Moesta et al. 2017), however, find that such jets are subject to instabilities unless the initial star is endowed with a (likely unrealistically large) pre-collapse field of $\sim 10^{13} \mathrm{G}$. If such instabilities set in, matter is exposed for longer to the central neutrino emission and therefore raises its $Y_{e}$ to large enough values to avoid significant platinum peak contributions. This interesting topic certainly warrants more work in the future. For now we conclude, that additional contributions at both the light and heavy $r$-process end are possible, but are - based on current numbers - not strictly required. It is fair to state, however, that the corresponding chemical galactic evolution questions are not yet fully understood and will require further studies.

Using the same method as described in Rosswog et al. (2017; their Sect. 3.4), we estimate the expected number of events like AT2017gfo that peak above a given limiting magnitude, see Fig. 7. For this, we used the blackbody model described in Kasliwal et al. (2017) and a reference event rate of $500 \mathrm{Gpc}^{-3} \mathrm{yr}^{-1}$. Due to the early blue peak of the observed transient, the expected numbers in the optical are large. A survey like ZTF ( $g<22$ mag 600-s exposures for GW follow up) could detect all NSMs with such a blue peak within the LIGO range, of which we would expect approximately one per year. With a larger optical survey telescope such as LSST 1000 macronovae could become observable per year. This, however, requires that the follow-up is triggered the same night because $g$-band fades rapidly and the numbers drop to only one event per year with 4 days after the merger. Observations at longer wavelengths would provide a larger window. In $i$-band, the number of observable macronovae 4 days after the merger is nearly two orders of magnitude larger. Similarly in the NIR, a 60-second exposure with VIRCAM in the $K$ band would be sufficient and the transient remains observable for more than a week.

The long awaited era of multi-messenger GW-astronomy has now finally begun and the first multi-messenger detection of a merging neutron star binary has conclusively proven the longheld conjectures of producing short GRBs and forging heavy elements, thereby providing the first directly observed constraints of rates and ejecta masses. How representative this first

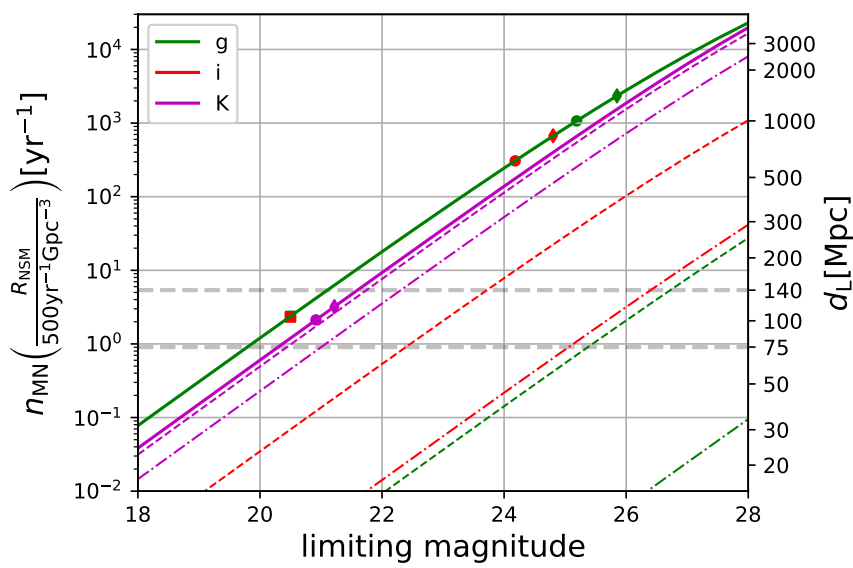

Fig. 7. Expected number of transients similar to AT2017gfo that peak above a given $g-, i$-, or $K$-band limiting magnitude. Calculations are based on the black-body model presented in Kasliwal et al. (2017). Solid lines are based on the peak brightness, while dashed and dashdotted lines are based on the brightness between 4 and 7 days after the merger, respectively. Note that the result for $i$-band at peak is not shown because it is practically the same as for $g$-band. The circles and diamonds correspond to the depths of 60- and 180-second exposures, respectively (for LSST in $g$ - and $i$-band and for VISTA in $K$-band). The square marker shows the depth of a ZTF 600-second exposure in the $g$-band. The dashed lines show assumed ranges for GW detections of $75 \mathrm{Mpc}$ for NSNS mergers and $140 \mathrm{Mpc}$ for NS-BH.

event was will have to be probed by future multi-messenger detections.

Acknowledgements. We thank the anonymous referee for her/his insightful comments that helped to improve our paper. It is a great pleasure to thank Friedrich-Karl Thielemann for insightful discussions. SR has been supported by the Swedish Research Council (VR) under grant number 2016-03657_3, by the Swedish National Space Board under grant number Dnr. 107/16. SR, JS and AG are supported by the research environment grant "Gravitational Radiation and Electromagnetic Astrophysical Transients (GREAT)" funded by the Swedish Research council (VR) under Dnr 2016- 06012. MMK acknowledges support by the GROWTH project funded by the National Science Foundation under PIRE Grant No 1545949. This work has further been supported by the CompStar network, COST Action MP1304. Some of the simulations were performed on the resources provided by the North-German Supercomputing Alliance (HLRN). A portion of this work was also carried out under the auspices of the National Nuclear Security Administration of the US Department of Energy at Los Alamos National Laboratory under Contract No. DE-AC52-06NA25396 (O.K., R.W.). Some of the simulations used in this work were performed on the resources provided by the Los Alamos National Laboratory Institutional Computing Program (O.K.,R.W.). OK and RW are thankful to Aimee L. Hungerford, Chris L. Fryer, and Christopher J. Fontes for inspiring and productive discussions.

\section{References}

Abadie, J., Abbott, B. P., Abbott, R., et al. 2010, Class. Quantum Grav., 27, 173001

Abbott, B. P., Abbott, R., Abbott, T. D., et al. 2017a, ApJ, 848, L12

Abbott, B. P., Abbott, R., Abbott, T. D., et al. 2017b, Nature, 551, 85

Abbott, B. P., Abbott, R., Abbott, T. D., et al. 2017c, ApJ, 848, L2

Abbott, B. P., Abbott, R., Abbott, T. D., et al. 2017d, Phys. Rev. Lett. 119, 161101

Arcavi, I., Hosseinzadeh, G., Howell, D. A., et al. 2017, Nature, 551, 64

Argast, D., Samland, M., Thielemann, F.-K., \& Qian, Y.-Z. 2004, A\&A, 416, 997

Barnes, J., \& Kasen, D. 2013, ApJ, 775, 18

Barnes, J., Kasen, D., Wu, M.-R., \& Martinez-Pinedo, G. 2016, ApJ, 829, 110

Bauswein, A., Ardevol Pulpillo, R., Janka, H.-T., \& Goriely, S. 2014, ApJ, 795, L9

Beloborodov, A. M., 2008, in Hyper-Accreting Black Holes, ed. M. Axelsson, AIP Conf. Ser., 1054, 51

Beniamini, P., Hotokezaka, K., \& Piran, T. 2016, ApJ, 832, 149 
Berger, E., Fong, W., \& Chornock, R. 2013, ApJ, 774, L23

Burbidge, E. M., Burbidge, G. R., Fowler, W. A., \& Hoyle, F. 1957, Rev. Mod. Phys., 29, 547

Cameron, A. G. W. 1957, Stellar evolution, nuclear astrophysics, and nucleogenesis, Chalk River Report, CRL-41

Chornock, R. Berger, E., Kasen, D., et al. 2017, ApJ, 848, L19

Ciolfi, R., \& Siegel, D. M. 2015, ApJ, 798, L36

Coulter, D. A., Foley, R. J. Kilpatrick, C. D., et al. 2017, Science, 358, 1556

Cowperthwaite, P. S., Berger, E., Villar, V. A., et al. 2017, ApJ, 848, L17

Dessart, L., Ott, C. D., Burrows, A., Rosswog, S., \& Livne, E. 2009, ApJ, 690, 1681

Duflo, J., \& Zuker, A. P. 1995, Phys. Rev. C, 52, R23

Eichler, D., Livio, M., Piran, T., \& Schramm, D. N. 1989, Nature, 340, 126

Evans, P., Cenko, S. B., Kennea, J. A., et al. 2017, Science, 358, 1565

Fernandez, R., \& Metzger, B. D. 2013, ApJ, 763, 108

Fernandez, R., Kasen, D., Metzger, B. D., \& Quataert, E. 2015, MNRAS, 446, 750

Freiburghaus, C., Rembges, J., Rauscher, T., et al. 1999a, ApJ, 516, 381

Freiburghaus, C., Rosswog, S., \& Thielemann, F.-K. 1999b, ApJ, 525, L121

Goldstein, A., Veres, P., Burns, E., et al. 2017, ApJ, 848, L14

Goriely, S., Bauswein, A., \& Janka, H.-T. 2011, ApJ, 738, L32

Grossman, D., Korobkin, O., Rosswog, S., \& Piran, T. 2014, MNRAS, 439 757

Guillochon, J., Parrent, J., Kelley, L. Z., \& Margutti, R. 2017, ApJ, 835, 64

Hallinan, G., Corsi, A., Mooley, K. P., et al. 2017, Science, 358, 1579

Hansen, T. T., Simon, J. D., Marshall, J. L., et al. 2017, ApJ, 838, 44

Hotokezaka, K., Kiuchi, K., Kyutoku, K., et al. 2013, Phys. Rev. D, 87, 2

Hotokezaka, K., Piran, T., \& Paul, M. 2015, Nat. Phys., 11, 1042

Hotokezaka, K., Sari, R., \& Piran, T. 2017, MNRAS, 468, 91

Hulse, R. A., \& Taylor, J. H. 1975, ApJ, 195, L51

Just, O., Bauswein, A., Pulpillo, R. A., Goriely, S., \& Janka, H.-T. 2015, MNRAS, 448, 541

Kasen, D., Badnell, N. R., \& Barnes, J. 2013, ApJ, 774, 25

Kasen, D., Fernandez, R., \& Metzger, B. D. 2015, MNRAS, 450, 1777

Kasen, D., Metzger, B. D., Barnes, J., Quataert, E., \& Ramirez-Ruiz, E. 2017, Nature, 551, 80

Kasliwal, M. M., Nakar, L., Singer, P., et al. 2017, Science, 358, 1559

Kim, C., Perera, B. B. P., \& McLaughlin, M. A. 2015, MNRAS, 448, 928

Korobkin, O., Rosswog, S., Arcones, A., \& Winteler, C. 2012, MNRAS, 426, 1940

Kulkarni, S. R. 2005, ArXiv e-prints [arXiv:astro-ph/0510256]

Lai, D. K., Bolte, M., Johnson, J. A., et al. 2008, ApJ, 681, 1524

Lattimer, J. M., \& Schramm, D. N. 1974, ApJ, 192, L145

Lee, W. H., \& Ramirez-Ruiz, E. 2007, New J. Phys., 9, 17

Levan, A., Lyman, J. D., Tanvir, N. R., et al. 2017, ApJ, 848, L28

Li, L.-X., \& Paczyński B. 1998, ApJ, 507, L59

Lyman, J. D., Lamb, G. P., Levan, A. J., et al. 2018, Nat. Astron., in press [arXiv: 1801.02669]

Margutti, R., Berger, E., Fong, W., et al. 2017, ApJ, 848, L20

Martin, D., Perego, A., Arcones, A., et al. 2015, ApJ, 813, 2

Matteucci, F., Romano, D., Arcones, A., Korobkin, O., \& Rosswog, S. 2014 MNRAS, 438, 2177
Mennekens, N., \& Vanbeveren, D. 2014, A\&A, 564, A134

Metzger, B. D., \& Fernandez, R. 2014, MNRAS, 441, 3444

Metzger, B. D., Piro, A. L., \& Quataert, E. 2008, MNRAS, 390, 781

Metzger, B. D., Martinez-Pinedo, G., Darbha, S., et al. 2010, MNRAS, 406, 2650

Möller, P., Nix, J. R., Myers, W. D., \& Swiatecki, W. J. 1995, At. Data Nucl. Data Tables, 59, 185

Moesta, P., Roberts, L. K., Halevi, G., et al. 2017, ArXiv e-prints [arXiv: 1712.09370]

Perego, A., Gafton, E., Cabezón, R., Rosswog, S., \& Liebendörfer, M. 2014a, A\&A, 568, A11

Perego, A., Rosswog, S., Cabezón, R. M., et al. 2014b, MNRAS, 443, 3134

Petrillo, C., Dietz, A., \& Cavaglia, M. 2013, ApJ, 767, 140

Pian, E., D'Avanzo, P., Benetti, S., et al. 2017, Nature, 551, 67

Pinto, P. A., \& Eastman, R. G. 2000, ApJ, 530, 744

Qian, Y. Z., \& Woosley, S. 1996, ApJ, 471, 331

Radice, D., Galeazzi, F., Lippuner, J., et al. 2016, MNRAS, 460, 3255

Roberts, L. F., Kasen, D., Lee, W. H., \& Ramirez-Ruiz, E. 2011, ApJ, 736, L21

Rosswog, S. 2005, ApJ, 634, 1202

Rosswog, S. 2013, Roy. Soc. London Philos. Trans. Ser. A, 371, 20272

Rosswog, S., Thielemann, F. K., Davies, M. B., Benz, W., \& Piran, T. 1998, in Coalescing Neutron Stars: A Solution to the $r$-process Problem? eds. W. Hillebrandt, \& E. Muller, Proc. of the 9th Workshop on Nuclear Astrophysics, 103

Rosswog, S., Liebendörfer, M., Thielemann, F.-K., et al. 1999, A\&A, 341, 499

Rosswog, S., Korobkin, O., Arcones, A., Thielemann, F.-K., \& Piran, T. 2014 MNRAS, 439, 744

Rosswog, S., Feindt, U., Korobkin, O., et al. 2017, Class. Quantum Grav., 34, 104001

Shen, S., Cooke, R. J., Ramirez-Ruiz, E., et al. 2015, ApJ, 807, 115

Siegel, D. M., \& Metzger, B. D. 2017, Phys. Rev. Lett., 119, 23,

Smartt, S. J., Chen, T.-W., Jerkstrand, A., et. al. 2017, Nature, 551, 75

Sneden, C., Cowan, J. J., \& Gallino, R. 2008, ARA\&A, 46, 241

Tanaka, M., \& Hotokezaka, K. 2013, ApJ, 775, 113

Tanvir, N. R., Levan, A. J., Fruchter, A. S., et al. 2013, Nature, 500, 547

Tanvir, N., Levan, A.J., González Fernández, C., et al. 2017, ApJ, 848, L27

Taylor, J. H., \& Weisberg, J. M. 1982, ApJ, 253, 908

Thielemann, F.-K., Arcones, A., Käppeli, R., et al. 2011, Prog. Part. Nucl. Phys., 66, 346

Thielemann, F.-K., Eichler, M., Panov, I. V. , \& Wehmeyer, B. 2017, Ann. Rev. Nucl. Part. Sci., 67, 253

van de Voort, F., Quataert, E., Hopkins, P. F., Keres, D., \& Faucher-Giguere, C.-A. 2015, MNRAS, 447, 140

Villar, V. A., Guillochon, J., Berger, E., et al. 2017, ApJ, 851, L21

Wallner, A., Faestermann, T., Feige, J., et al. 2015, Nat. Commun., 6, 5956

Wanajo, S., \& Janka, H.-T. 2012, ApJ, 746, 180

Wanajo, S., Sekiguchi, Y., Nishimura, N., et al. 2014, ApJ, 789, L39

Winteler, C. 2012, Ph.D. Thesis, University Basel, CH

Winteler, C., Käppeli, R., Perego, A., et al. 2012, ApJ, 750, L22

Wollaeger, R. T., Korobkin, O., Fontes, C. J., et al. 2018, MNRAS, 478, 3298

Wu, M.-R., Fernandez, R., Martinez-Pinedo, G., \& Metzger, B. D. 2016, MNRAS, 463, 2323 


\section{Appendix A: Summary of the semi-analytic model}

Here we briefly summarize the major ingredients of our semianalytic macronova model. It uses the analytic density structure found from solving the spherical Euler equations for a self-similar homologous flow that is derived in Sect. 2.1.1 of Wollaeger et al. (2018). To extract the radiative signature, it makes use of the analytic solution of the comoving frame transport equations for constant opacity as derived by Pinto \& Eastman (2000) in the context of type Ia supernovae. This solution has been carefully cross-checked against a multigroup radiative transfer code, see Sect. 2.3.1 of Wollaeger et al. (2018).

The heating rates $\dot{q}$ that enter the model can be chosen to be either a) an analytic prescription, as Eq. (4) in Korobkin et al. (2012) or b) be taken as the output of a nuclear reaction network calculation. In all calculations shown in this paper we use the output of a nuclear reation network calculation performed with WinNet (Winteler 2012; Winteler et al. 2012) and the FRDM mass model. For the heating efficiencies $f_{\text {tot }}$ we use the timedependent averages of all the FRDM models shown Rosswog et al. (2017), which, in turn, are based on the work of Barnes et al. (2016). As discussed in the main text, Sect. 2, we mimick the results of the Duflo-Zuker mass formula by multiplying the net heating rates $\left(\dot{q} f_{\text {tot }}\right)$ by a factor of 5 for those cases where nuclei heavier than lead are formed.

For our expansion model we follow Wollaeger et al. (2018) and start from the mass and momentum equations of ideal, non-relativistic hydrodynamics for a sphere that expands into vacuum. For self-similar homologous flow a scale parameter $R(t)$ and a shape function $\varphi(x)$ can be introduced, $x$ being the dimensionless radial coordinate $x=r / R(t)$, so that density and velocity are

$\rho(t, r)=\frac{\varphi(x)}{R(t)^{3}} \quad$ and $\quad v(t, r)=r \frac{\dot{R}(t)}{R(t)}$.

If a polytropic equation of state with $\Gamma=4 / 3$ ("radiation dominated flow") is used, the Euler equations admit a closed form solution:

$\varphi(x)=\rho_{0} R_{0}^{3}\left(1-x^{2}\right)^{3}$

and

$\left(t-t_{0}\right)=\frac{R(t)}{v_{\max }} \sqrt{1-\frac{R_{0}}{R(t)}}+\frac{R_{0}}{v_{\max }} \log \left[\frac{R(t)}{R_{0}}\left(1-\sqrt{1-\frac{R_{0}}{R(t)}}\right)^{2}\right]$,

where $R_{0}=R\left(t_{0}\right), \rho_{0}$ the initial central density and $v_{\max }$ the expansion velocity. For $t \gg t_{0}$ Eq. (A.3) reduces to $R(t) \simeq v_{\max } t$ and the density profile becomes

$\rho(t, r)=\rho_{0}\left(\frac{t_{0}}{t}\right)^{3}\left(1-\frac{r^{2}}{v_{\max }^{2} t^{2}}\right)^{3}$

where $v_{\max }$ is the expansion front velocity. The ejecta mass and average velocity are

$m_{\mathrm{ej}}=\frac{64 \pi}{315} \rho_{0} t_{0}^{3} v_{\max }^{3} \quad$ and $\quad \bar{v}=\frac{63}{128} v_{\max }$.

Coming from a radioactively heated, initially optically thick cloud of matter, macronova emission bears some similarity with type Ia supernovae. Since the energy injection decreases rapidly with time, the light curve will peak as soon as the injected energy has a chance to escape being converted into kinetic energy. This happens when the diffusion time becomes comparable to the elapsed time. In our model, we extract the radiative signature based on an eigenmode expansion formalism developed by Pinto \& Eastman (2000). Our notation and details of derivation follow Wollaeger et al. (2018), where it was applied in the context of macronova with uniform density (see their Appendix A). Here we only summarize the main points, see the original paper for the justification of the physical assumptions and for the detailed derivation.

The starting point is the semi-relativistic diffusion equation which for radiation dominated flows reads

$\frac{\mathrm{D} E}{\mathrm{D} t}-\nabla \cdot\left(\frac{c}{3 \kappa \rho} \nabla E\right)+\frac{4}{3} E \nabla \cdot \boldsymbol{v}=\rho \dot{q}(t)$,

where $E$ is the internal energy density, D/D $t$ the Lagrangian time derivative and $\kappa$ the constant (gray) opacity. Using dimensionless quantities and assuming spherical symmetry and homologous expansion one finds

$\frac{\mathrm{D} E}{\mathrm{D} t}-\frac{1}{R^{2} x^{2}}\left[\frac{c}{3 \kappa \rho} x^{2} E^{\prime}\right]^{\prime}+\frac{4 E}{t}=\rho \dot{q}(t)$,

where the primed quantities are being differentiated with respect to $x$. The second term in Eq. (A.6) is the divergence of the radiative diffusion flux $\boldsymbol{F}$ :

$\boldsymbol{F}=-\frac{c}{3 \kappa \rho} \nabla E \equiv-\frac{c}{3 \chi} \nabla E$,

where we introducted the extinction coefficient $\chi \equiv \kappa \rho$. With a subsequent separation of variables in mind, we make the Ansatz

$E(x, t)=E_{0}\left[\frac{t_{0}}{t}\right]^{4} \psi(x) \phi(t), \quad$ and $\quad \rho(x, t)=\rho_{0}\left[\frac{t_{0}}{t}\right]^{3} \varphi(x)$.

and recast the Eq. (A.7) into the following form:

$\frac{t_{0}}{t} \psi \dot{\phi}-\phi \frac{1}{\tau_{0} x^{2}}\left[\frac{x^{2} \psi^{\prime}}{\varphi}\right]^{\prime}=\frac{\rho_{0} \dot{q}(t)}{E_{0}} \varphi$,

where we introduced the timescale $\tau_{0} \equiv \frac{3 \kappa \rho_{0} R_{0}^{2}}{c}$.

It is convenient to use the rescaled time coordinate $\zeta \equiv t / t_{0}$ with $\bar{\tau}_{0} \equiv \tau / t_{0}$ :

$\psi \frac{\partial \phi}{\partial \zeta}-\zeta \phi \frac{1}{\bar{\tau}_{0} x^{2}}\left[\frac{x^{2} \psi^{\prime}}{\varphi}\right]^{\prime}=\frac{\rho_{0} t_{0} \dot{q}(\zeta)}{E_{0}} \varphi$

The corresponding homogeneous linear equation,

$\frac{1}{\zeta \phi(\zeta)} \frac{\partial \phi(\zeta)}{\partial \zeta}-\frac{1}{\bar{\tau}_{0} \psi(x) x^{2}}\left[\frac{x^{2} \psi(x)^{\prime}}{\varphi(x)}\right]^{\prime}=0$

admits a separation of variables for some constant $\lambda$ :

$\frac{\bar{\tau}_{0}}{\zeta \phi(\zeta)} \frac{\partial \phi(\zeta)}{\partial \zeta}=-\lambda$,
$\left[\frac{x^{2} \psi(x)^{\prime}}{\varphi(x)}\right]^{\prime}+\lambda x^{2} \psi(x)=0$.

Equation (A.14) is an eigenvalue problem. We can now make a substitution $\psi(x) \rightarrow\left(1-x^{2}\right)^{4} z(x)$ to regularize it at the outer 

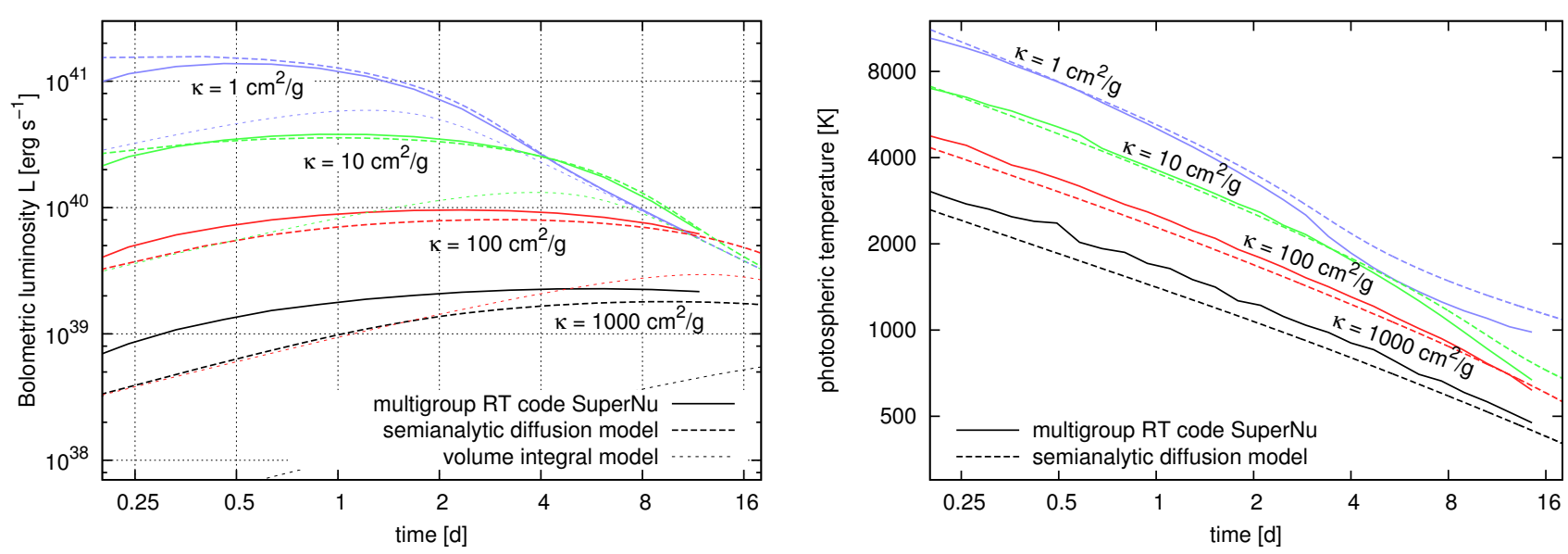

Fig. A.1. Left: Bolometric luminosity for the described semianalytic diffusion model (dashed lines) with opacities $\kappa=1,10,100,1000 \mathrm{~cm}^{2} \mathrm{~g}^{-1}$ compared against full multigroup Monte Carlo radiative transfer models (solid lines) and the substantially simpler model of Grossman et al. (2014) that uses volume integration over the radiative zone ("volume integral model"). Right: Comparison of the photospheric temperature evolution between our semianalytic diffusion model (dashed) with the radiative transfer code SuperNu (solid).

boundary, where density becomes zero. The power 4 is motivated by the following reasoning: for an adiabatic radiation-dominated outflow with a constant entropy $T^{3} / \rho=$ const. the temperature profile should be $\propto\left(1-x^{2}\right)$, provided that the density profile is $\propto\left(1-x^{2}\right)^{3}$. Correspondingly, the internal energy density $E \propto$ $T^{4} \propto\left(1-x^{2}\right)^{4}$. form:

The eigenvalue problem can be cast into Sturm-Liouville

$$
\frac{\mathrm{d}}{\mathrm{d} x}\left[x^{2}\left(1-x^{2}\right)^{5} \frac{\mathrm{d} z}{\mathrm{~d} x}\right]+x^{2}\left(1-x^{2}\right)^{4}\left[\lambda\left(1-x^{2}\right)^{4}-24\right] z=0,
$$

for which there exists a spectrum of distinct real eigenvalues $\left\{\lambda_{m}\right\}$ and an orthogonal basis $\left\{z_{m}(x)\right\}$ in Hilbert space with respect to the scalar product

$\langle f \mid g\rangle \equiv \int_{0}^{1} x^{2}\left(1-x^{2}\right)^{8} f(x) g(x) \mathrm{d} x$.

This is a well-posed eigenvalue problem which can be solved using a variety of numerical methods. We use a Galerkin method with linear finite element discretization on a uniform grid with $N=100$ points. Having computed the eigenvalues and eigenfunctions, we can expand a solution to the inhomogeneous problem Eq. (A.11) in eigenfunctions with time-dependent expansion coefficients $\phi_{m}(\zeta)$ :

$E(t, x)=E_{0} \zeta^{-4} \sum_{m} \phi_{m}(\zeta) \psi_{m}(x)$

where the functions $\psi_{m}(x) \equiv\left(1-x^{2}\right)^{4} z_{m}(x)$ are weighted eigenfunctions of Eq. (A.15). Equation (A.13) splits into a series of decoupled first-order ODEs for the functions $\phi_{m}(\zeta)$ :

$\left(\frac{d \phi_{m}}{d \zeta}+\frac{\zeta}{\bar{\tau}_{0}} \phi_{m} \lambda_{m}\right) N_{m}^{2}=\frac{\rho_{0} t_{0} \dot{q}(\zeta)}{E_{0}} \zeta \mathrm{d}_{m}$,

where

$N_{m}^{2} \equiv \int_{0}^{1} x^{2} \psi_{m}^{2}(x) \mathrm{d} x \equiv \int_{0}^{1} x^{2}\left(1-x^{2}\right)^{8} z_{m}^{2}(x) \mathrm{d} x$,

and

$d_{m} \equiv \int_{0}^{1} x^{2} \varphi(x) \psi_{m}(x) \mathrm{d} x \equiv \int_{0}^{1} x^{2}\left(1-x^{2}\right)^{7} z_{m}(x) \mathrm{d} x$
We solve these equations numerically with a CrankNicholson integrator.

The bolometric luminosity is proportional to the flux Eq. (A.8) through the outer boundary at $x=1$ :

$$
\begin{aligned}
(t) & =\left.4 \pi R(t)^{2} \cdot\left(-\frac{c}{3 \kappa \rho} \cdot \frac{\partial E}{\partial r}\right)\right|_{x=1} \\
& =-\left.\frac{4 \pi c R_{0} E_{0}}{3 \kappa \rho} \sum_{m=1}^{N} \phi_{m}\left(\frac{t}{t_{0}}\right) \psi_{m}^{\prime}(x)\right|_{x=1} \\
& =\left.\frac{32 \pi c R_{0} E_{0}}{3 \kappa \rho_{0}} \sum_{m=1}^{N} \phi_{m}\left(\frac{t}{t_{0}}\right) \cdot z_{m}(x)\right|_{x=1},
\end{aligned}
$$

where we used the following simplification to express the derivative at $x=1$ :

$$
\left.\frac{\psi_{m}^{\prime}(x)}{\rho(x)}\right|_{x=1}=\left.\frac{\left[\left(1-x^{2}\right)^{4} z_{m}(x)\right]^{\prime}}{\rho_{0}\left(1-x^{2}\right)^{3}}\right|_{x=1}=-\left.8 z_{m}(x)\right|_{x=1} .
$$

To scrutinize our approach, we have computed light curves for a range of opacities $\kappa=1,10,100,1000 \mathrm{~cm}^{2} \mathrm{~g}^{-1}$ and a powerlaw heating rate $\dot{q}(t)=q_{0} t_{d}^{\alpha}$ with $q_{0}=5 \times 10^{9} \mathrm{erg} \mathrm{g}^{-1} \mathrm{~s}^{-1}$ and $\alpha=-1.3$. Figure A.1 presents the comparison of bolometric light curves caluclated with the described approach and the corresponding one from the full multigroup Monte Carlo radiative transfer code SuperNu ${ }^{6}$. The semianalytic diffusion model performs substantially better than the simpler analytic solution of Grossman et al. (2014), which uses integration of the energy rate over the radiative zone outside of the trapped region.

As demonstrated in Wollaeger et al. (2018), the spectrum of gray opacity models is well approximated by a blackbody with effective temperature at the photosphere:

$T_{\mathrm{eff}}(t)=\sqrt[4]{\frac{L(t)}{4 \pi \sigma R_{\mathrm{ph}}(t)^{2}}}$.

The right panel in A.1 shows the effective photospheric temperature evolution for full radiative transfer models and the temperatures for semianalytic models, computed using expression (A.25) and assuming the photosphere at optical depth $\tau_{\mathrm{ph}}=2 / 3$.

6 https://bitbucket.org/drrossum/supernu/wiki/Home 